\title{
RESEARCH
}

Open Access

\section{Phylogenetic analysis of the Tc1/mariner superfamily reveals the unexplored diversity of pogo-like elements}

\author{
Mathilde Dupeyron ${ }^{*}$, Tobias Baril, Chris Bass and Alexander Hayward ${ }^{*}$
}

\begin{abstract}
Background: TC1/mariner transposons are widespread DNA transposable elements (TEs) that have made important contributions to the evolution of host genomic complexity in metazoans. However, the evolution and diversity of the Tc1/mariner superfamily remains poorly understood. Following recent developments in genome sequencing and the availability of a wealth of new genomes, Tc1/mariner TEs have been identified in many new taxa across the eukaryotic tree of life. To date, the majority of studies focussing on Tc1/mariner elements have considered only a single host lineage or just a small number of host lineages. Thus, much remains to be learnt about the evolution of TC1/mariner TEs by performing analyses that consider elements that originate from across host diversity.

Results: We mined the non-redundant database of NCBI using BLASTp searches, with transposase sequences from a diverse set of reference Tc1/mariner elements as queries. A total of $5158 \mathrm{Tc} /$ mariner elements were retrieved and used to reconstruct evolutionary relationships within the superfamily. The resulting phylogeny is well resolved and includes several new groups of Tc1/mariner elements. In particular, we identify a new family of plant-genome restricted Tc1/mariner elements, which we call PlantMar. We also show that the pogo family is much larger and more diverse than previously appreciated, and we review evidence for a potential revision of its status to become a separate superfamily.
\end{abstract}

Conclusions: Our study provides an overview of Tc1-mariner phylogeny and summarises the impressive diversity of Tc1-mariner TEs among sequenced eukaryotes. Tc1/mariner TEs are successful in a wide range of eukaryotes, especially unikonts (the taxonomic supergroup containing Amoebozoa, Opisthokonta, Breviatea, and Apusomonadida). In particular, ecdysozoa, and especially arthropods, emerge as important hosts for Tc1/mariner elements (except the PlantMar family). Meanwhile, the pogo family, which is by far the largest Tc1/mariner family, also includes many elements from fungal and chordate genomes. Moreover, there is evidence of the repeated exaptation of pogo elements in vertebrates, including humans, in addition to the well-known example of CENP-B. Collectively, our findings provide a considerable advancement in understanding of Tc1/mariner elements, and more generally they suggest that much work remains to improve understanding of the diversity and evolution of DNA TES.

Keywords: Transposase, DNA transposon, Transposable element, Evolution, Phylogeny, Tigger

\footnotetext{
*Correspondence: m.dupeyron@exeter.ac.uk; alex.hayward@exeter.ac.uk Centre for Ecology and Conservation, University of Exeter, Penryn Campus, Penryn, Cornwall TR10 9FE, UK
}

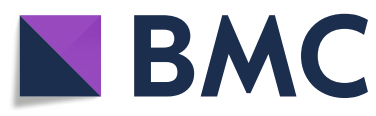

(c) The Author(s). 2020 Open Access This article is licensed under a Creative Commons Attribution 4.0 International License, which permits use, sharing, adaptation, distribution and reproduction in any medium or format, as long as you give appropriate credit to the original author(s) and the source, provide a link to the Creative Commons licence, and indicate if changes were made. The images or other third party material in this article are included in the article's Creative Commons licence, unless indicated otherwise in a credit line to the material. If material is not included in the article's Creative Commons licence and your intended use is not permitted by statutory regulation or exceeds the permitted use, you will need to obtain permission directly from the copyright holder. To view a copy of this licence, visit http://creativecommons.org/licenses/by/4.0/. The Creative Commons Public Domain Dedication waiver (http://creativecommons.org/publicdomain/zero/1.0/) applies to the data made available in this article, unless otherwise stated in a credit line to the data. 


\section{Introduction}

DNA transposable elements (TEs) or 'class II elements' are a major category of repetitive DNA. DNA TEs use a cut-and-paste mechanism catalysed by a transposase enzyme to mobilize within the host genome, and may comprise a considerable proportion of total host genomic DNA [1-3]. DNA TEs typically contain a transposase domain enclosed by terminal inverted repeats (TIRs). However, this organisation is flexible and TEs may contain additional ORFs or motifs, and in some cases they can lack TIRs. TIRs act as recognition sites for the transposase enzyme, which excises the transposon and relocates it to a new position within the genome during transposition.

The Tc1/mariner superfamily is an important group of DNA TEs discovered in invertebrate genomes during the early 1980s, and is considered to be the most widespread DNA TE superfamily among eukaryotes [4]. The first Tc1/mariner element discovered was Tc1, during examination of restriction fragment strain polymorphisms in the nematode roundworm Caenorhabditis elegans in 1983 [5]. Three years later, the mariner element was identified in the fruitfly Drosophila mauritiana during study of the white-peach $\left(w^{\text {pch }}\right)$ eye colour mutant [6]. In 1990, a bacterial insertion sequence found in the Shigella genome, IS630, was linked to Tc1, as it shares a TA target site duplication (TSD) formed after successful transposition [7]. At first considered to represent different families, Tc1, mariner, and IS630 were later gathered together as the IS630/Tc1/mariner (ITm) group, based on their shared mode of transposition via a DNA intermediate, their TA target site, and transposase sequence homology $[8,9]$. Soon after, the pogo family was also classified as a member of ITm [8]. Pogo was characterised in the genome of the fruitfly $D$. melanogaster, during a study of the promoter region of the white locus, where two insertions, one of them a pogo element, caused the white-eosin $\left(w^{e}\right)$ mutation [10]. In the late 1990s, mariner-like elements were discovered in plant genomes, starting with soybean [11].

In total, eight families are currently included within the ITm group, which are classified according to the number of amino acid residues present between the second and third aspartic acid residue (D), or the second aspartic acid and the glutamic acid residue (E) of the transposase catalytic domain (i.e. DDD/E) [12]. The eight described ITm families are: mariner (DD34D), Tc1 (DD34E), pogo (DDxD), DD39D from plants, DD37E from mosquitoes, $D D 37 D$ from insects and nematodes, $D D 34 E$ from ciliates, and the bacteria insertion sequence group IS630 [12, 13]. However, the branch linking the $D D 34 E$ family to the other families is poorly supported, and its membership to the group is considered questionable (Fig. 4 in [12]). Furthermore, bacterial IS630 sequences are only distantly related to eukaryotic DNA TEs and are not considered to be similar to eukaryotic DNA TEs. Thus, here we restrict our focus to eukaryotic Tc1/mariner elements, and consider IS630 as the outgroup to the Tc1/mariner superfamily.

The Tc1/mariner superfamily is well known due to the widespread use of several Tc1/mariner elements as genetic tools. For example, Sleeping Beauty $(S B)$ is a synthetic TE reconstructed from multiple inactive fish Tc1like transposon sequences, that is widely used in genetic engineering for somatic gene delivery and functional genomics (e.g. gene discovery) [14]. Similarly, a Tc1-like element found in the Rana pipiens genome called Frog Prince was reconstructed for gene-trapping in fish, amphibians and mammals [15]. The first mariner element to be used as a genetic tool was Hsmarl, which was reconstructed from the human genome [16]. Hsmar1 transposes efficiently in vertebrate cells and has been linked to the formation of non-autonomous MITE elements, making it a useful system to study the transposition dynamics and evolution of mariner elements in primate genomes [17]. Additionally, the fungal transposon Fot1 of the pogo family is used as a tagging system to study the regulation of gene expression in fungi [18].

Adding to the fame of Tc1/mariner elements, the superfamily includes several high profile examples of the molecular domestication of transposon sequences for host genomic purposes. For example, SETMAR is a chimeric gene that is expressed in most cells and tissues in anthropoid primates, which has roles in key processes such as DNA methylation, repair and alternative splicing [19]. SETMAR is composed of a SET gene, shared among vertebrates, and an Hsmarl transposase. The transposase is flanked by a $3^{\prime}$ TIR and an Alu retrotransposon on the $5^{\prime}$ end, and transposition is estimated to have occurred 40-58 million years ago in an ancestral lineage of the anthropoid primates [20]. Another important example of the molecular domestication of a Tc1/ mariner element is centromere protein $B(C E N P-B)$, a conserved protein found in mammalian centromeres [21]. CENP-B appears to have been domesticated from a Tigger-like element (from the pogo family), which belongs to a group of Tc1/mariner elements that contain a CENP-B box in their $5^{\prime}$ TIR [21]. The CENP-B protein binds the $C E N P-B$ box which, in addition to being located in Tigger-like elements, is also located in host alpha-satellite centromeric DNA [22], and is thought to be involved in kinetochore formation (although its exact role in centromere functioning remains unclear) [23].

Despite the importance, diversity, and very large host range of Tc1/mariner elements, there are no recent studies of their evolution and classification. Several publications report the diversity of a subset of Tc1/mariner elements from the genomes of a focal group of 
organisms [24-27]. However, the most comprehensive phylogenetic analysis of the evolution of the Tc1/mariner superfamily, including most known elements of each family and clade support values, was published in 2001 [12]. Here we employ the many new Tc1/mariner sequences detected in recently sequenced eukaryote genomes to perform a large-scale phylogenetic analysis of the Tc1/mariner superfamily, which we use to examine the evolution, diversity, and classification of the group.

\section{Results and discussion}

We recovered 5158 Tc1/mariner elements from the genomes of 922 species from across eukaryotic diversity, adding greatly to the known diversity of the Tc1/mariner superfamily (Additional file 1). Based on the results of our phylogenetic analyses (Fig. 1, Additional File 2), we present the evolutionary relationships among eight families that form the Tc1/mariner superfamily. We identify four previously characterised major families, each of which contain a large number of sequences: Tc1: 1009 sequences; mariner: 938 sequences; PlantMar (formerly referred to as ' $D D 39 D$ '): 542 sequences; pogo: 2620 sequences, and four minor families that contain just a few sequences each: Tec: 4 sequences; TBE: 16 sequences; DD37E(L31): 11 sequences; HvSm: 4 sequences (Fig. 1, Additional File 2). The four minor families include three previously described families (i.e. $D D 37 E(L 31), T e c, T B E$ ) and one new family, which we name $\mathrm{HvSm}$, after its host species (Hydra vulgaris and Schmidtea mediterranea).

We show that Tc1/Mariner phylogeny is composed of two main clades, one containing the Tc1, mariner and PlantMar families plus the four minor families, and one entirely composed of the pogo family. The pogo family is separated from the other Tc1/mariner families by a relatively long branch, indicating considerable evolutionary distinctness. Further, we reveal that pogo contains a very large number of elements (more than double the remaining Tc1/Mariner elements), which were isolated from the genomes of a diverse array of host taxa, and these elements feature a helix-turn-helix and CENP-B-

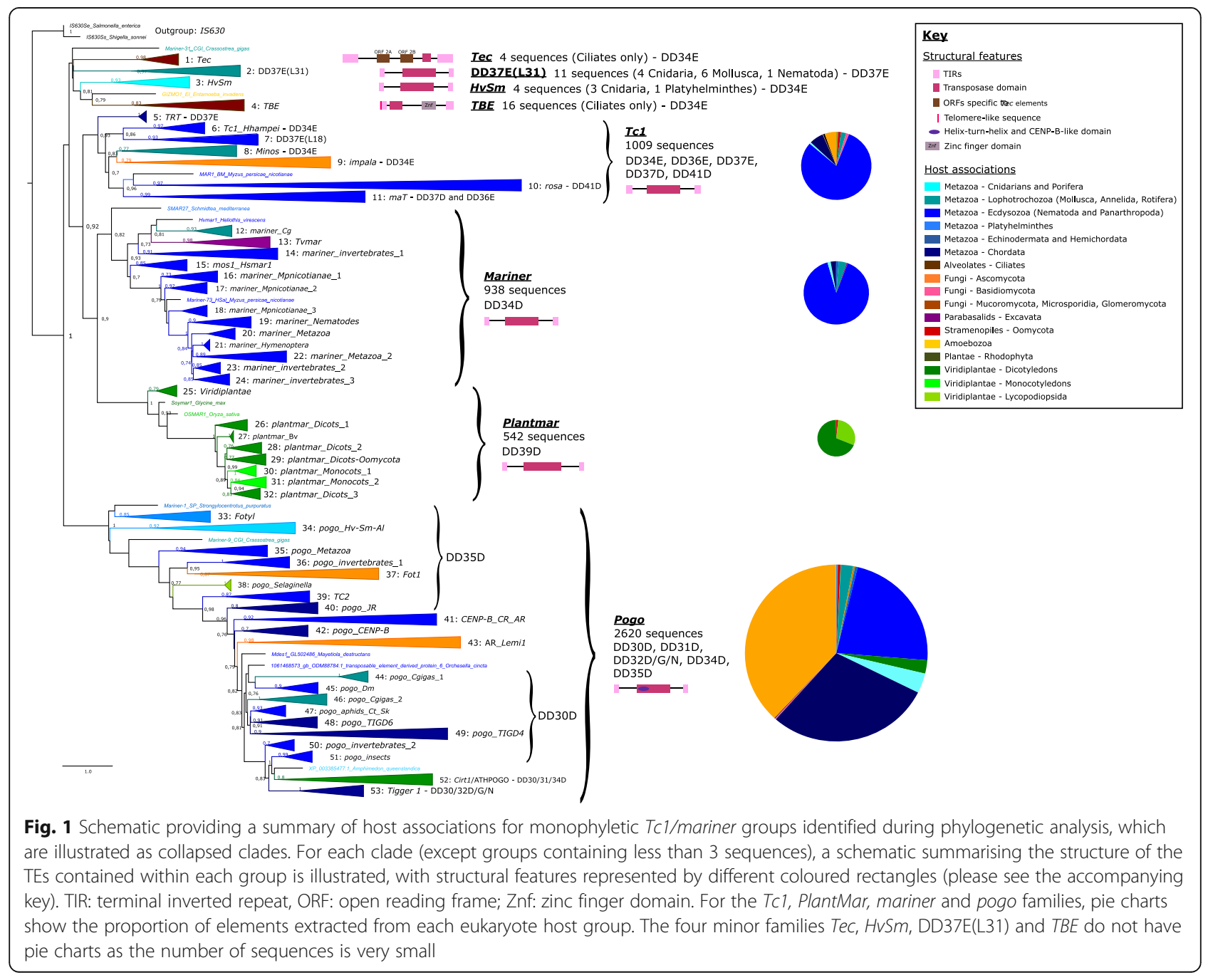


like domain. Taking these features into account, we suggest below that pogo may be more appropriately classified as its own superfamily.

The main Tc1/mariner clade consists of a poorlysupported grouping, which unites four minor families that share a sister-group relationship with a wellsupported clade containing the Tc1, mariner, and PlantMar families. The host taxonomic origin of the main Tc1/mariner clade is unclear, but there is clear evidence of a single transition to plant hosts leading to the origin of the PlantMar family (groups 25-32). Meanwhile, more basal subclades in the pogo clade contain DNA TEs of metazoan origin, suggesting an origin in animals (possibly flatworms or cnidarians), with two independent transitions to plant genomes: a small clade involving the club moss Selaginella (Clade 38), and a larger clade containing a number of dicotyledonous plant hosts (Clade 52).

Several isolated taxa consisting of a single transposase sequence are scattered across Tc1/mariner phylogeny (Fig. 1). These sequences typically occur at the end of relatively long branches, and it is possible that they are sole representatives of additional families, which will become clearer in the future as genomic data continue to accumulate. Below we discuss the major patterns observed in the Tc1/mariner phylogeny in more detail, alongside accompanying insights gained about the evolution of this large and important DNA TE superfamily.

\section{The $T c 1$ family}

The Tc1 family is well-supported with 93\% bootstrap support, and is composed of seven subfamilies (Fig. 1). The majority of subfamilies within $T c 1$ have $\geq 85 \%$ bootstrap support, although the Minos and impala subfamilies have 77 and $79 \%$ support respectively (Fig. 1). Subfamilies are named in accordance with previously described groups or their main host genomes (Fig. 1).

Tc1 elements share a similar structure, with TIRs of $30 \mathrm{bp}$, a single ORF encoding a transposase, and a mean length of $\sim 1300 \mathrm{bp}[9,28,29]$. However, some Tc1 elements have long TIRs of several hundred base pairs, such as Minos [30, 31]. We confirm the DDD/E structure identified previously for the different $T c 1$ families: TRT (Group 5) is DD37E, Tc1_Hhampei (Group 6) and Minos (Group 8) are DD34E, impala (Group 9) is DD37E, rosa (Group 10) is DD41D, and maT (Group 11 ) is DD37D (Additional file 3A). We identify a large number of newly identified sequences from host genomes including arthropods, fungi, archeaplastids, oomycetes and several bacteria (in black, Additional file 2 ). The apparent presence of a very small number of TEs apparently isolated from bacterial genomes within the otherwise eukaryote-restricted Tc1/mariner superfamily suggests either their horizontal transfer across major domains of life, or that contamination events have occurred (and bacterial host associations are spurious). To attempt to discriminate between these alternatives and confirm integration in a bacterial genomic context, we searched for flanking sequences in the corresponding elements in Genbank. Either no flanking sequence was present (i.e. only partial TE sequence existed without TIRs), or only very short up- or downstream sequences were present. Thus, we suggest that in the absence of evidence for horizontal transmission, it is prudent to consider these sequences as contamination or erroneously labelled with regard to their origin.

This study confirms the success of $T c 1$ elements in a diverse range of organisms, but especially ecdysozoan metazoans (i.e. arthropods and nematodes) (Additional file 2). The impressive number of sequences recovered from the spider Stegodyphus mimosarum (Clade 11, Additional file 2), together with a pattern of very short terminal branch lengths, suggest these elements underwent a recent expansion or are still undergoing a significant burst of activity in this taxon. The little brown bat, Myotis lucifugus, is the only mammalian vertebrate identified by our analyses that possesses Tc1 sequences (Clade 11, Additional file 2). The M. lucifugus genome is of particular interest, because in contrast to other mammalian genomes, it contains recently active DNA TEs [1]. Given that M. lucifugus feeds on a very wide range of arthropods (at least 61 insect species and 5 spider species [32]), its diet may increase its exposure to TEs from arthropods and the likelihood of horizontal transmission. The S. mimosarum and M. lucifugus Tc1 sequences are present in the maT subfamily (Clade 11, Fig. 1), which contains elements from an unusual grouping of hosts including red algae, oomycetes, basidiomycetes, mycorrhizal fungi, and microsporidians (Additional file 2). Such a patchy taxonomic distribution, together with the parasitic lifestyle of several of these host organisms, suggests a history of horizontal transfer in this group [33]. For example, parasitic organisms such as oomycetes may facilitate horizontal transfer, since they are widespread pathogens that share an intimate association with their hosts, they occupy varied environments, and they infect an extremely large host range [34]. Further study is required to elucidate the relationships between similar $T c 1$ elements shared by extremely divergent host taxa, and addressing this question will become more straightforward as genomic data from a wider sampling of host diversity accumulates.

A recent study of Tc1/mariner elements in the genome of the Pacific oyster Crassostrea gigas identified a new subfamily and a new family of elements. Specifically, these new groups were described as a: (i) a Mariner-18_ CGi-like group, $D D 37 E(L 18)$, which appeared to form a new subfamily within the Tc1 family; and, (ii) a Mariner- 
31_CGi-like group, $D D 37 E(L 31)$, that appeared to form a whole new family more basal to pogo [35]. We included the sequences from this analysis in our sequence alignment (Additional file 4), and found that: (i) the $D D 37 E(L 18)$ subfamily is present in the Tc1 family, but unfortunately it does not group together with Mariner18_CGi (the C. gigas sequence it was named after) which occurs in Minos (Group 8, Additional File 2); while, (ii) the $D D 37 E(L 31)$ family shares a sister-group relationship with the Tec subfamily, and again unfortunately does not group together with Mariner-31_CGi, which groups as an isolated sequence with the minor families Tec, $D D 37 E(L 31), H \nu S m$ and TBE (Fig. 1, Additional file 2). This situation highlights the difficulties associated with describing new TE families on the basis of single taxon studies, and the problematic nomenclature that can arise as a consequence.

\section{The mariner family}

Mariner is a very well known family of Tc1/mariner elements, and previous studies have revealed its wide distribution among metazoans, partly as a consequence of horizontal transmission [36]. Most mariner elements are short transposons of $\sim 1200-1300 \mathrm{bp}$, that contain a single ORF encoding a DD34D transposase (Additional file 4), with short $\sim 20$-30 bp TIRs.

Our analysis demonstrates that mariner elements are present primarily in arthropod genomes, and we identify a great many more elements than previously known. Nevertheless, several elements in the mariner clade also originate from the genomes of a diverse range of other taxa (i.e. excavates, platyhelminths, gastropods, nematodes, and rotifers). In total, we update the number of mariner subfamilies to 13 (Additional File 2).

Previous studies have tended to focus on mariner elements from particular host groups, for example hydra and flatworms [37] or aphids [27]. Where applicable, we maintain the names adopted for previously identified subfamilies in our phylogeny, but in some cases elements representing these groups are located in multiple subfamilies together with numerous sequences from invertebrate genomes, and we have renamed them accordingly (Additional File 2). These differences result from the greater host diversity considered here and the many numerous new sequences we identify in the mariner family. Thus, while studies focussing on single host taxa can be useful to identify new TE diversity within a particular host genome, going forward we suggest a cautious approach toward suggesting new families, based on broad scale analyses that consider a wide range of host taxonomic diversity only.

A small number of elements that are labelled as originating from bacterial host genomes are also present within the mariner family, but we could not find any evidence to suggest horizontal transfer, leading us to conclude that these sequences most likely represent labelling errors or contamination, as in the $T c 1$ clade.

\section{The PlantMar family}

The few studies that have analysed sequences from the Tc1/mariner DD39D group typically included them as members of the mariner family [11,38]. In contrast, our analysis facilitated the retrieval of many additional sequences from this group, which almost all originate from plant genomes. We find strong evidence for the existence of a separate plant Tc1/mariner family that we name 'PlantMar' (Clades 25-32, Fig. 1). The PlantMar family forms a monophyletic group with full bootstrap support that originates at the end of a long-branch, with a sister-group relationship to the mariner family.

The structure of PlantMar elements is typical for Tc1/ mariner, with short TIRs, an overall length of $2-6 \mathrm{~kb}$, and a DD39D transposase domain amino acid structure (Additional File 4). The PlantMar family is restricted to dicotyledonous and monocotyledonous plant hosts, and a small number of oomycete hosts [39]. We identified 8 subfamilies in the PlantMar family, with elements generally clustering according to whether their host plant is mono- or dicotyledonous (Fig. 1, Additional file 2). None of the elements in the PlantMar family belong to early plant phyla such as Glaucophytes (freshwater microscopic algae), Rhodophyta (red algae), Zygnematales (green algae) or Bryophyta (liverworts, hornworts and mosses), leading us to formulate two alternative hypotheses, either these elements: (i) underwent horizontal transfer to angiosperm genomes, most likely from an ancestral Tc1/mariner element present in Oomycota, fungal pathogens and/or viruses; or, (ii) were once present in Archaeplastida and were subsequently lost in earlybranching phyla, remaining present in higher Viridiplantae genomes only. Given the host taxonomic context present in the closely related $T c 1$ and mariner families (i.e. a widespread distribution across arthropods), hypothesis (i) appears is more likely, suggesting horizontal transfer followed by a host switch, leading to the origin of a distinct family restricted to plants.

Interestingly, as with evolution of the Mutator DNA TEs [40], once a host switch to plants occurred, the ability to switch back to other branches of eukaryotic life seems to have been almost completely lost. With the exception of a few sequences from Oomycota, we find no transitions back to non-plant hosts within the PlantMar clade. Given that there are now at least two examples of DNA TEs making strict unidirectional switches onto plant hosts, it will be interesting to examine if this pattern is repeated across a wider swathe of DNA TE diversity, and to investigate the mechanisms that prevent a 
switch back from plants to a wider diversity of hosts in these cases.

\section{$T B E$ and Tec from ciliates}

Two small Tc1/mariner subfamilies are restricted to ciliates: Tec and TBE (Clades 1 and 4, Fig. 1, Additional file 2 ). We maintained the names previously used in the literature for both subfamilies [41, 42]. TBE (telomere bearing elements) is named for the presence of telomere-like sequences at the tips of the TIRs, and the $T B E$ clade is sister to the GIZMO element identified from the amoeba Entameoba invadens [41]. Elements in the TBE family have small $\sim 80 \mathrm{bp}$ TIRs and they carry three ORFs: a transposase, a small ORF of unknown function, and a zinc finger protein [43].

Tec elements have a highly unusual structure for DNA transposons, with very long TIRs of $\sim 700 \mathrm{bp}$ and three ORFs, one of them in complement [42]. Despite these differences from typical Tc1/mariner transposons, Tec and TBE encode a transposase containing a DD34E motif in the third ORF [44]. Moreover, Tec elements carry a site-specific recombinase in the second ORF which can perform transposition in the absence of a dedicated transposase [45]. This is consistent with the very short third ORF that carries the transposase, which may be inactive.

Ciliates are peculiar eukaryotes with cells containing two nuclei: a micronucleus containing the germline, which is mostly transcriptionally inactive and has a TE content of $\sim 20 \%$, and a somatic transcriptionally active macronucleus without repeats [46]. During formation of the macronucleus, the non-coding part of the micronuclear DNA is deleted, including the transposon content, leading to the formation of 'internal eliminated sequences' (IESs), with TSD-like sequences corresponding to TE remnants [43]. The precise mechanisms of macronucleus formation and TBE and Tec element transposition remain unclear. A signature of purifying selection detected for ORFs present in TBE elements suggests that the excision activity of the transposase may have been harnessed by the host genome, for example in the elimination process of non-coding sequences during macronucleus formation [42, 43]. The peculiar life cycle of ciliates may explain some of the unusual structural differences exhibited by TBE and Tec elements relative to Tc1/mariner TEs present in the genomes of other eukaryotes.

\section{HvSm - a new family with only four sequences}

Three sequences from the freshwater cnidarian polyp Hydra vulgaris, and one sequence from the platyhelminth Schmidtea mediterranea form a new family that we name ' $H v S m$ ', reflecting its main host association (Clade 3, Fig. 1 and Additional file 2). Surprisingly,
$\mathrm{HvSm}$ does not contain sequences from other cnidarian or platyhelminth species, despite the presence of $26 \mathrm{cni}$ darian genomes and 36 flatworm genomes in Genbank. Nevertheless, this remains a relatively small number of genomes compared to estimates of the total number of described platyhelminthes (>18,000 [47]) and cnidarians (>9000 [48]), and we anticipate that more elements will be identified in this family.

We analysed the structure of the four elements in $\mathrm{HvSm}$ and found that one of them is partial, containing a $579 \mathrm{bp}$ transposase ORF, but no TIRs. The full-length copies contain $585 \mathrm{bp}, 756 \mathrm{bp}$ and $1106 \mathrm{bp}$ transposases, with $15 \mathrm{bp}, 20 \mathrm{bp}$ and $56 \mathrm{bp}$ TIRs flanked by TA TSDs, and have an overall length of $1716 \mathrm{bp}, 1841 \mathrm{bp}$ and 2751 $\mathrm{bp}$, respectively (two full-length sequences are provided in Additional file 5). Each transposase shows a DD34E motif, similar to the closely related Tec and TBE families, but differentiating them from the $D D 37 E(L 31)$ family.

\section{The pogo family}

The most recent consideration of the pogo family was in 2014, which included 60 sequences from the genomes of 38 host species, belonging to 3 kingdoms of eukaryotic life [49]. In contrast, we have retrieved 2620 pogo-like sequences from the genomes of 519 host species, belonging to six kingdoms of eukaryotic life (Fig. 1). Contrary to the Tc1 and mariner families, which occur predominantly in ecdysozoan genomes, pogo elements are also found in many ascomycete fungus and chordate genomes (Fig. 1). Thus, we reveal that the pogo family is dispersed across a considerably wider diversity of hosts than previously appreciated. Additionally, we find that pogo is the largest Tc1/mariner family, containing more than half the total number of all Tc1/mariner elements recovered in our study (Fig. 1 and Additional file 2).

Notably, we identified distinct DDD motifs in the transposase domains of pogo elements: groups 33 to 40 display a DD35D pattern; groups 44 to 51 display a DD30D pattern; while groups 52 and 53 show a varying pattern of DD30-32D (Fig. 1 and Additional file 3B). This reveals new information about the structure of the pogo transposase catalytic domain, contrasting with what has been described previously. Groups 41 to 43 do not show a specific pattern, with sequences displaying DD30D, DD31D or DD34D motifs, with no apparently dominant type.

Taking the range of new evidence into account, we suggest that pogo may be more appropriately classified as an independent superfamily of DNA TEs, instead of a family within the Tc1/mariner superfamily. This evidence includes: the relatively long branch length leading to the pogo clade, its distinct pattern of host associations, the size of the pogo family, its sister group relationship 
to a clade containing all other Tc1/mariner elements, the distinct transposase structure of pogo elements where the transposase domain contains a helix-turnhelix and CENP-B-like domain, and the distinct pattern of DDD motifs in the catalytic domain of pogo transposases.

Previous studies revealed the presence of pogo-like elements in mammals, reptiles, fish, insects, nematodes, molluscs, fungi and plants $[8,18,35,49,50]$, and classified them in five main groups: TC2 (human and fish), AR (plants and fungi), JR (metazoans), CR (metazoans) and Fot1 (fungi) [22, 49]. Our phylogenetic analysis indicates the presence of 21 pogo subfamilies (Fig. 1 and Additional file 2), most of which contain sequences from a single host kingdom (e.g. metazoans or fungi, Fig. 1, Additional File 2). Several previously described groups (e.g. AR, JR and CR [22]) are not monophyletic in our phylogeny, and the sequences from these groups are instead scattered across various pogo subfamilies (Fig. 1 and Additional file 2). More basal subgroups contain invertebrate sequences and many fungal sequences, especially from Fusarium, from which Fot1 was described (Clade 37, Fig. 1 and Additional file 2). Below we provide a short description of several notable subfamilies, drawing attention to particular points of interest.

Clade 33 is composed of a small set of sequences originating from diverse organisms, such as Amoebozoa, Fungi, and Echinodermata. Several small subgroupings contain sequences from distantly related terrestrial and aquatic organisms, resulting in a diverse and somewhat puzzling host distribution pattern. Meanwhile, the large Fot1 subfamily consists mainly of fungal sequences, however, one subgroup contains six sequences from the $\mathrm{Pa}-$ cific oyster Crassostrea gigas. Oysters live in aquatic marine environments, whereas fungal species containing pogo-like elements that group closely to oyster Fot1-like elements are terrestrial. Clade 38 is composed of only two sequences from the lycophyte plant Selaginella moellendorffii. This model species is a primitive vascular plant and is of interest because it is a very ancient group [51]. S. moellendorffii has one of the smallest genomes known among plants and more than a third of its genome is composed of TEs [52]. The detection of two pogo-like sequences in such an early vascular plant genome presents two hypotheses: (i) pogo elements are ancient DNA TEs that were present in early eukaryote taxa, and have subsequently undergone elimination in most plant lineages, but were successfully retained in many unikonts (i.e. Metazoa and Fungi); or alternatively, (ii) pogo elements underwent multiple independent horizontal transfer events to plant genomes, leading to their presence in several dicotyledonous plant genomes and $S$. moellendorffii. Further research into the activity of pogolike elements in early plant taxa is needed to clarify their evolution in this group. TC2 elements were originally described in the puffer fish, Takifugu rubripes, and the human genome (Clade 39, Fig.1 and Additional file 2). We find closely related TC2-like elements from a diverse host range in our phylogeny: Helobdella robusta a leech species, various vertebrates (fish, gecko, snake and lemur), a mite, a beetle, and many sequences from the spider Stegodyphus mimosarum. Either this pattern is a result of the presence of the ancestral element in the common ancestor of Bilateria, followed by loss in most bilaterian lineages, or a consequence of repeated horizontal transfer events.

We identify considerable confusion surrounding the classification of Tigger elements. The first Tigger elements, Tigger 1 and Tigger 2 , were isolated from mammalian genomes, and were described with reference to their similarity to CENP-B and pogo elements more widely $[10,21]$. Tigger 1 was specifically classified as a 'mammalian pogo' [10], which we confirm here. However, we reveal that Tigger1 (Clade 53, Additional file 2), and Tigger2 (Clade 40, Additional file 2) are separated by considerable phylogenetic distance in our analysis. Thus, while the transposase sequences of Tigger 1 and Tigger 2 are similar, the availability of a much larger number of pogo-like elements now demonstrates that Tigger 1 and Tigger 2 are relatively distantly related within a wider evolutionary context. Further, since the original canonical pogo element occurs in a clade situated between Tigger1 and Tigger2 (Clade 45, Additional file 2), it is apparent that Tigger elements are polyphyletic, and do not form a distinct monophyletic group. Additionally, over time the use of sequence similarity to classify elements has led to the annotation of new Tigger-like elements across a large swathe of pogo-like element diversity (Clade 40, 43, 49, 52, and 53, Additional file 2), and we suggest that this practice is abandoned in favour of phylogenetic approaches.

\section{Domestication of pogo elements}

Pogo transposases are known to have been exapted for host functions in metazoan genomes, with a well-known example being the evolution of centromeric protein $C E N P-B$ [22]. We find evidence for additional domestications in several other pogo lineages. Specifically, we provide evidence of exaptation for seven pogo-like elements, which are frequently referred to as 'Tigger transposable element derived' genes (TIGD1-TIGD7) [53], in tetrapod host genomes, especially mammals.

We checked the genomic context of the human TIGD6 gene in Ensembl [54] and identified the nearest upstream and downstream genes: SLC26A2, a solute carrier transporter, and $H M G X B 3$, a DNA binding protein. We then used the orthology verification tool in Ensembl using the human TIGD6, SLC26A2 and HMGXB3 genes 
as queries to identify conservation in their arrangement in the genomes of other mammal species (Additional file 11). Similarly, the seven TIGD genes all show syntenic organisation in mammals and other vertebrates (reptiles, amphibians and birds) (Additional files 6, 7, 8, 9, 10, 11 and 12). The shared genomic organisation of the TIGDlike elements in vertebrate genomes suggests an ancient insertion event in an ancestral vertebrate. Detailed information for each of these TIGD-like elements and their genomic environment is provided in Additional files 6, $7,8,9,10,11$ and 12 .

TIGD1-TIGD7 display full-length ORF sequences corresponding to the transposase domain, suggesting conservation of functionality, and likely exaptation for host genomic purposes. In Homo, BioGrid [55] lists 3 protein-protein interactions for TIGD1, 3 for TIGD2, 4 for TIGD3, 3 for TIGD4, 50 for TIGD5, 21 for TIGD6, and 5 for TIGD7. Thus, there is good experimental evidence that TIGD proteins often interact with large numbers of host proteins, suggesting an embedded role for TIGD genes in the host genome context. Further, the Bgee [56] and Genevisible [57] gene expression databases suggest that TIGD genes are widely expressed in Homo: TIGD1-168 organs, with highest expression in the brain and immune cells; TIGD2-178 organs, with highest expression in the placenta; TIGD3-63 organs, with highest expression in the cerebellar hemisphere, blood and leukocytes; TIGD4-103 organs, with highest expression in sperm and the testes; TIGD5-203 organs, with highest expression in the quadriceps femoris muscle, the deltoid muscle, the parotid gland, and the epithelium of the nasal cavity and kidney; TIGD6-136 organs, with highest expression in the prostate gland, spinal cord, and across the endocrine system; and TIGD7-175 organs, with highest expression in the testis.

Considering the pattern of divergent TIGD-like sequences in our phylogenetic tree (Additional file 2), we performed a NCBI BLASTp search focussing on TIGDlike sequences, using coding sequence corresponding to the ancestral transposase domain of each TIGD1-7 gene as queries. We then performed a phylogenetic analysis on the retrieved sequences to examine TIGD diversity in more detail (alignment: Additional file 13, tree: Additional file 14). Below we briefly summarise the major patterns present in the TIGD tree and discuss their implications.

TIGD-like sequences occur in highly supported clades $(\geq 97 \%)$ and are restricted to tetrapod hosts and their immediate relatives (i.e. the Coelacanth lobe-finned fish, Latimeria chalumnae) (Additional file 14). In several cases, closely related sequences from invertebrate hosts occur more basally to TIGD clades (i.e. TIGD1, TIGD3, TIGD4, TIGD6), suggesting multiple independent domestication events of different ancestral pogo elements
(Additional file 14). In contrast, the TIGD2, TIGD5, and TIGD7 clades are united together in a group, and it is possible that these genes may represent paralogues (i.e. be descended by gene-duplication from a single pogo domestication event). The host distribution of TIGD sequences within tetrapods remains patchy. Additionally, while sequences in several TIGD clades are widely distributed across tetrapod diversity (TIGD1, TIGD4, TIGD5), the taxonomic distribution of others are more narrow (for example, no sequences were identified from birds for TIGD3), or are either partially restricted to mammals (TIGD2) or are entirely restricted to mammals (TIGD6, TIGD7). These findings suggest that either TIGD genes have been selectively retained in certain host lineages following an ancient origin pre-dating the tetrapods, or that sequences in certain host lineages have become too divergent for our current approach to recover. Detailed work to distinguish between these alternatives would be valuable to further illuminate TIGD evolution in the future.

The TIGD catalytic motif shows considerable variation, presumably as a consequence of positive selection following exaptation to optimise TIGD proteins for new roles in the host genome: TIGD1 is DD32D; TIGD2 is mostly DD34S; TIGD3 is DA30P in mammals, DA35P in birds, and DD33H in reptiles; TIGD4 is DD30K or DE30K in vertebrates, except birds where we could not identify the third position; TIGD5 is DA60E in mammals, DS/T48E in birds, and DN33D in reptiles; TIGD6 is mostly DD30N; and TIGD7 is DD34N (Additional file $3 \mathrm{~B}$ and $\mathrm{C}$ ).

The evidence discussed above suggests that TIGD genes may play important fundamental roles in vertebrates and the group deserves closer research attention. We are conscious that our search will not have uncovered all Tigger-like elements, and intensive study of TIGD genes, particularly involving validation in the lab, will likely yield considerable further insights into their domestication and roles.

\section{Host range evolution and horizontal transfer}

The majority of Tc1/mariner elements were recovered from ecdysozoan host genomes (i.e. arthropods and nematodes). However, host range often varies among families. Most dramatically, the PlantMar family contains elements from plants and stramenopiles only. Meanwhile, the pogo family includes large proportions of elements that originate from fungus and chordate genomes (Fig. 1). Interestingly, despite a relatively cosmopolitan distribution across eukaryotes, relatively few Tc1/mariner elements are present in more basal eukaryote lineages, for example, we identified just two elements in amoeboid protists (Amoebozoa), and no elements in green algae (Chlorophyta). 
Very little is known about the mechanisms that underlie host range in TEs, and the patterns we observe for Tc1/mariner elements are no exception. For example, whether host range is primarily driven by encounter or by compatibility filers, sensu theory from host-parasite interactions [58], remains very much an open research question.

A tanglegram indicating links between Tc1/mariner phylogeny and host phylogeny at the level of eukaryote orders is presented in Fig. 2. The tanglegram illustrates a high level of incongruence, suggesting widespread horizontal transfer of Tc1/mariner TEs across host diversity. An alternative explanation for the observed pattern is that each Tc1/mariner family was present in the eukaryotic ancestral lineage, and subsequently active elements representing each Tc1/mariner family have been selectively retained in just some host lineages. However, this would require invoking a very large number of loss events across eukaryote phylogeny. Consequently, given the unlikeliness of the alternative hypothesis together with recent research demonstrating the frequency with which horizontal transmission can occur (see below), we suggest that a history of horizontal transfer is the most likely explanation for the observed host distribution of Tc1/mariner elements.

Over recent years, considerable evidence of the widespread horizontal transfer of TEs (HTTs) has become apparent $[33,59,60]$. Of particular relevance is a recent analysis of HTT among 195 insect genomes, which found that Tc1/mariner elements were the most frequently horizontally transmitted TE group [60]. Indeed, despite their highly conservative approach, the authors identified > 1000 putative horizontal transfer events involving Tc1/mariner elements among a relatively small sample of insect genomes [60], equating to approximately 5 HTTs per insect species considered. Moreover, Tc1/mariner elements were also found to occupy the highest mean fraction of the host genome among horizontally transferred TE groups [60], adding evidence to their propensity to transfer horizontally.

Confirming recent examples of HTT based on complete transposase sequences remains problematic. We identified 15 potential cases of recent HTTs in the Tc1/mariner superfamily, based on strong clade support values between two or more sequences present in the genomes of distantly related eukaryotes (Table 1). For

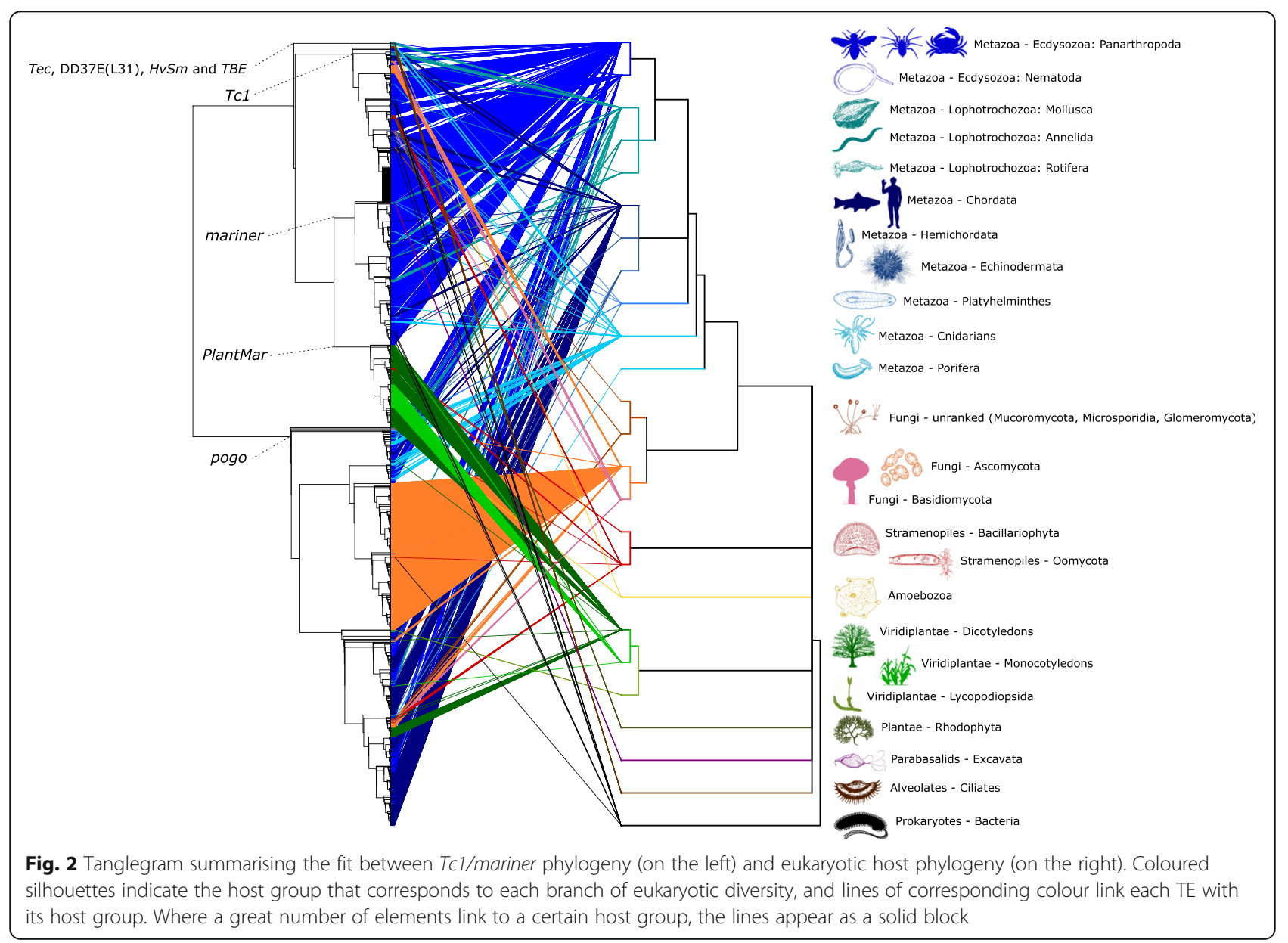


example, in the mariner family, a sequence from the sheep Ovis aries, clusters with sequences from the ant Oocera biroi, and the bacterium Pseudomonas monteilii (Clade 32, Additional file 2). Ecologically, it is plausible to invoke a HTT event between these taxa given their shared environment. However, as we were unable to confirm the host genomic context of the transposases in question, we cannot rule out other explanations for the observed patterns (such as contamination during lab work or sequencing). In the pogo family, we identified a potential HTT between the blue tit (Cyanistes caeruleus) and the pepper (Capsicum annuum) (Clade 52, Additional file 2), and the lettuce (Latuca sativa) and the yellow sugar cane aphid (Sipha flava) (Clade 50, Additional file 2). In both cases, we searched for full-length elements in each genome to check flanking sequences (see Methods). However, once more, in both cases the element or partial element was located on a small contig, making verification impossible (Table 1 ). We were able to find a full-length element in just three cases of potential HTT, which involved the following host genomes: the White-Ruffed Manakin bird (Corapipo altera) and the squinting bush brown butterfly (Bicyclus anynana); the Queensland fruit fly (Bactrocera tryoni) and the bacterium Desulfovibrio; and, the Chinese tree shrew (Tupaia chinensis) and several hymenopterans (Table 1).

For each case of potential HTT we identified, if a genome was available and we were able to identify a full transposase domain, we performed an NCBI BLASTn search of the host genome using the transposase domain of the element as the query. In five cases out of seven, this identified just one hit for the TE involved, suggesting that the sequence may result from contamination rather than represent a real transposon present in that host genome. In the two cases where $>1$ copy of the transposase was found, the identity of each hit was > $95 \%$ in one genome, but $<95 \%$ in the other species, suggesting its presence in one species may be in doubt. Thus, no convincing cases of very recent HTT could be identified. As more high quality long-read genomes become available for interrogation, our ability to identify recent HTT events will increase, and the Tc1/mariner superfamily represents an excellent model for further study.

\section{Conclusions}

Tc1/mariner is a widespread DNA TE superfamily that is especially common in fungal and animal hosts. Four major Tc1/mariner families dominate the superfamily (Tc1, mariner, PlantMar, pogo), while four minor families contain just a few sequences each ( Tec, $\mathrm{HvSm}$, DD37E(L31), TBE). The well-known Tc1 and mariner families are well-supported, closely phylogenetically related, and found predominantly in invertebrate genomes. The PlantMar family is the sister-group to the mariner family, and host usage in this group indicates a strict switch from invertebrates to angiosperms. Pogo is by far the largest Tc1/Mariner family and displays the widest host distribution, with a large number of sequences from fungal and animal genomes, and a smaller number of sequences from plant, oomycete and amoeboid protist genomes (Figs. 1 and 2). We report several lines of evidence that suggest pogo may be more appropriately considered to be a separate DNA TE superfamily. Additionally, we find compelling evidence that pogo elements have been exapted by tetrapod genomes, and we provide

Table 1: Potential cases of horizontal transfer and the shared amino acid and nucleotide identity of transposases

\begin{tabular}{|c|c|c|c|c|c|c|}
\hline Species 1 & Full-length & $\begin{array}{c}\% \text { ID } \\
\text { transposase } \\
\text { (protein) }\end{array}$ & $\begin{array}{c}\text { \% ID full-length } \\
\text { element } \\
\text { (nucleotides) }\end{array}$ & Species 2 & Full-length & TE family \\
\hline Corapipo altera & Yes & 96,5 & 71 & Bicyclus anynana & Yes & mariner \\
\hline Desulfovibrio sp. & Yes & 73,51 & 69,6 & Bactrocera tryoni & Yes & mariner \\
\hline Bactrocera tryoni & Yes & 79,77 & 75,8 & Camponotus floridanus & Yes & mariner \\
\hline Camponotus floridanus & Yes & 72,85 & 70,8 & Desulfovibrio $s p$. & Yes & mariner \\
\hline Pseudomonas monteilii & No TIRs & 90,6 & $85,2^{*}$ & Ooceraea biroi & Yes & mariner \\
\hline Ooceraea biroi & Yes & 62,28 & 68,2 & Ovis aries & Yes & mariner \\
\hline Ovis aries & Yes & 65,5 & $60,9 *$ & Pseudomonas monteilii & No TIRs & mariner \\
\hline Tupaia chinensis & Yes & 78 & 67,7 & Pseudomyrmex gracilis & Yes & mariner \\
\hline Tatumella sp. & No & 73,2 & - & several arthropod species & No & $T c 1-\operatorname{ros} a$ \\
\hline Klebsiella pneumoniae & - & 85,51 & - & Diabrotica virgifera & No TIRs & $T c 1-m a T$ \\
\hline Desulfovibrio sp. & No TIRs & 93 & $58,91^{*}$ & Bombyx mori & Yes & $T c 1-m a T$ \\
\hline Acinetobacter baumannii - 3 sequences & - & 81,79 to 98,27 & - & Lepidopterans (Bombyx, Hypocoscoma) & No TIRs & $T c 1-m a T$ \\
\hline Bacillus thuringiensis - 2 sequences & No & 52,5 & - & Hydra vulgaris & No & $T c 1-m a T$ \\
\hline Lactuca sativa & No & 59,63 & - & Melanaphis sacchari & Yes & pogo \\
\hline Hymenobacter sp. & No & 83,7 & - & Parastagonospora nodorum & Yes & pogo \\
\hline Beijerinckia mobilis & No TIRs & 83,9 & $78^{*}$ & Aspergillus nidulans (x10) & Yes & pogo \\
\hline Capsicum baccatum & - & 88,75 & - & Cyanistes caeruleus & No TIRs & pogo \\
\hline Lactuca sativa & Almost & 96,8 & $88,7^{*}$ & Sipha flava (x2) in fact $A$. pisum & Yes & pogo \\
\hline
\end{tabular}

*only the transposase was used 
evidence that support the molecular domestication of TIGD1-TIGD7.

Several questions arise from our study, relating to the evolution and host range of Tc1/mariner elements. A key question is what enables Tc1/mariner elements to exploit such a diverse variety of hosts? For example, does their typically short and simple structure assist in their propensity to persist in the genomes of diverse host organisms, or do they possess a currently unidentified mechanism that facilitates host generalism? Additionally, what processes explain the variability of TIRs in Tc1/ mariner elements, and is this in some way related to their host-parasite dynamics? The extent to which Tc1/ mariner elements have been domesticated by host genomes, especially pogo elements, also remains unclear. Further research is required to clarify potential host shifts among distantly related host taxa, and it remains to be determined whether Tc1/mariner elements occasionally invade bacterial genomes or if their apparent presence in several bacteria can be decisively attributed to contamination. Meanwhile, a taxonomic issue is whether the pogo family would be better elevated to superfamily status, given the differences that set it apart from other Tc1/mariner elements.

\section{Methods}

\section{Mining and alignment of Tc1/mariner elements}

We employed BLASTp queries of the NCBI $n r$ database and a PSI-BLAST [61] of the swissprot database [62], using an in-house pipeline. Our query sequences were the Tc1/mariner DDD/E transposase domains provided in the supplementary material of Yuan \& Wessler [4], and sequences for Tc1/mariner transposons described in specific relevant publications $[1,12,22,24-27,35,49$, 63, 64], all in amino acid format and from seven Tc1/ mariner families. We focus on amino acid data since amino acid sequences evolve more slowly than DNA sequence data, facilitating sequence alignment at deeper evolutionary timescales, such as those across an entire DNA TE superfamily. This is important not just for phylogenetic accuracy, but also for interpreting patterns in transposase structure, such as diagnostic features of the DDD/E motif.

A preliminary phylogeny was estimated using query sequences together with those downloaded from the database search, after which one sequence was used as an additional query for any newly identified clades. A pipeline involving a filtering step to selectively retain matches with a minimum of $50 \%$ identity over at least $50 \%$ of the length of the query sequence was utilised. We manually added sequences from the studies of Ray et al. (2008) for Myotis lucifugus, Dupeyron et al. (2014) for Armadillidium vulgare, Puzakov et al. (2018) for Crassostrea gigas $[1,35,65]$, and sequences resulting from an independent DNA TEs annotation in Myzus persicae nicotianae (Toby Baril, unpublished data). In total, 5158 Tc1/mariner amino acid transposases were retrieved. Matches were extracted and processed into fasta format with the awk and sed EMBOSS tool v6.6.0.0 [66]. These hits correspond to transposase domains, and they do not reflect copy number in the respective host genomes from which they were retrieved, neither do they provide any indication of the abundance of nonautonomous elements in these genomes.

To briefly check the copy number of Tc1/mariner elements in eukaryote genomes, we used reference mariner and pogo elements described in Hydra vulgaris (Mariner-16_HM and Mariner-18_HM, respectively) to perform BLASTn searches on the WGS data of this species in NCBI. Two Whole Genome Sequence (WGS) projects are available in NCBI and with a similar quality, so we selected only one of them (ACZU00000000) to avoid hit duplication. The hit tables were downloaded and we counted full-length, partial and MITE copies as follows: full-length copies were considered when a scaffold hit showed at least $95 \%$ identity over at least $95 \%$ of the length of the element, partial copies when the hit was at least $80 \%$ identity over at least $50 \%$ of the length of the element, non-autonomous copies when the hit was at least $50 \%$ identity over at least $25 \%$ of the element length, and MITEs were inferred when the hit was at least 95\% identity over both the TIRs (according to hit coordinates). The summary of the copy numbers identified following this test can be found in Additional file 10 . We assessed the number of amino acid residues between the second $\mathrm{D}$ and the third $\mathrm{D} / \mathrm{E}$ in the transposase DDD/E motifs of each Tc1/mariner family, in our amino acid alignment in MEGA7 [67].

\section{Phylogenetic analyses}

We focussed on the transposase domain, since this is a highly conserved region among DNA TEs and there is an established precedent for using this region for higher level phylogenetic analyses in DNA TEs [4]. Transposase domains were aligned using the DDE domain alignment of Yuan \& Wessler [4] as a basis, and the profile alignment option of MUSCLE [68].

To infer the evolutionary history of the Tc1/mariner superfamily, we used FastTree v2.1.11 [69], which applies minimum-evolution subtree-pruning-regrafting (SPRs) and maximum-likelihood nearest-neighbor interchanges (NNIs). We used the -spr 4 option to improve SPRs, the '-mlacc 2' and '-slownni' options to increase accuracy, and we performed 1000 bootstrap repetitions. We used members of the IS630 TE group as an outgroup to root our phylogeny, since this group is considered most closely related to Tc1/mariner elements [4]. 
The tips of our tree were colour labelled by host taxon according to major taxonomic groupings in FigTree v1.4 (http://tree.bio.ed.ac.uk/software/figtree/). One element per superfamily and elements from newly identified groups were annotated with Artemis software [70], using the ORFfinder and BLASTx tools on NCBI for conserved domains, and the palindrome analyser tool of DNA Analyser to find TIRs [71]. Retrieval of classification information for the host species was computed with ETE3 [72] using in house python scripts. The tanglegram was produced using RStudio v3.5.1 Tydiverse [73] and ape v5.3 [74].

\section{Inferences of synteny}

Conservation of the genomic location of transposases and neighbouring genes constitutes evidence of synteny. Considering the apparent conservation of three pogo-like elements in vertebrate organisms in subgroups 48,49 and 53 (Additional file 2), we searched for their location and evidence of orthology in genomes available in Ensembl [54], using human sequences as a queries. We downloaded the tables provided by the orthology information contained on the website. According to the Homo sapiens genomic environment for each pogo sequence, we searched for orthology information for genes that were upstream and downstream of the transposase sequence. Summary tables were downloaded and manually checked, and the RStudio Tydiverse package was used to merge each table by the species column to provide a location for each TIGD element, and upstream and downstream genes.

\section{Study of potential horizontally transferred elements}

Potential horizontal transfer events were studied as follows: firstly, the amino acid percentage identity of the DDE domain was calculated using stretcher from EMBOSS tools [66]. Then, we searched for the nucleotide sequence of the elements involved in NCBI and the percentage identity of the DNA transposase sequence was also calculated. In three cases, we found the full-length element in both species involved, and we calculated the percentage identity of the whole element between each pair (Table 1). Following these steps, we searched for the element with the transposase nucleotide sequence as a query in the genome of the species' involved, if available in NCBI, to attempt to detect other copies. Only two pairs of species potentially involved in HTTs showed more than one hit for this search. However, the percentage identity was $<92 \%$ for these copies, and the overall percentage identity between the full-length elements in both species was $<80 \%$, so we did not pursue this further.

\section{Supplementary information}

Supplementary information accompanies this paper at https://doi.org/10. 1186/s13100-020-00212-0.

Additional file 1. Taxonomic information for each eukaryote host represented in the phylogenetic tree

Additional file 2. Phylogenetic tree of the amino-acid DDD/E transposase domain of $5158 \mathrm{Tc} /$ mariner elements. The tree results from a phylogenetic analysis using maximum likelihood inference, with 1000 bootstrap repetitions. Clade support values above $70 \%$ are indicated adjacent to each clade. Clades are divided into groups, with a corresponding clade name and number to the right. Elements are named according to their Repbase or Genbank ID, or according to the name provided in the article describing them. The host genome for each element is indicated to the right hand side of its ID, and labels are coloured broadly according to the taxonomic kingdom and class that the host species belongs to: shades of blue for metazoans, purple for excavates (Parabasalids), dark red for oomycetes (Stramenopiles), yellow for amoebozoans, shades of orange for fungi (pink for Basidiomycota), and shades of green for plants.

Additional file 3. Alignment text files showing the DDD/E structure of each clade showing a conserved amino acid residues number between the second $D$ and the third $D$ or the $E$ of the transposase domain. A) DDD/E alignment caption for HvSm and PlantMar. B) DDD alignment caption for TIGD1-4. C) DDD alignment caption for TIGD5-7.

Additional file 4. The amino acid alignment used to perform our phylogenetic analysis.

Additional file 5. Fasta sequences of the 75 full-length $T c 1 /$ mariner elements used in this study.

Additional files 6 to $\mathbf{1 2}$. Conserved locations of TIGD1 to TIGD7 in host vertebrate species and information about the upstream and downstream genes flanking them, retrieved from Ensembl [54]. Negative numbers indicate that the considered gene is upstream of the TIGD element.

Additional File 13. The amino acid alignment used to perform the TIGD1-TIGD7 phylogenetic analysis.

Additional File 14. Phylogenetic tree of the amino-acid DDD/E transposase domain of TIGD-like elements. The tree results from a phylogenetic analysis using maximum likelihood inference, with 1000 bootstrap repetitions. Clades are divided into groups, with a corresponding clade name and number to the right. The host genome for each element is indicated to the right hand side of its ID, and labels are coloured broadly according to the taxonomic kingdom and class that the host species belongs to: shades of blue for metazoans, purple for excavates (Parabasalids), dark red for oomycetes (Stramenopiles), yellow for amoebozoans, shades of orange for fungi (pink for Basidiomycota), and shades of green for plants.

Additional File 15. Table showing the results of assessing the copy number of full-length mariner and pogo elements in the Hydra vulgaris genome.

\section{Abbreviations}

TEs: Transposable elements; TIRs: Terminal inverted repeats; IESs: Internal eliminated sequences; TSD: Target site duplication; ITm: IS630-TC1/mariner; CENP-B: Centromere protein B; ORF: Open reading frame; MITEs: Miniature inverted-repeat transposable elements

\section{Acknowledgements}

Myzus cerasi and Acyrthosiphon pisum DNA sequencing data were downloaded from AphidBase. Funding for the sequencing was provided by ERC Starting Grant APHIDHOST-310190 awarded to Jorunn Bos at the James Hutton Institute, United Kingdom. Myzus persicae DNA sequencing data were also downloaded from AphidBase. Funding for Myzus persicae clone G006 genomic sequencing was provided by USDA-NIFA award 2010-65105-20558. Funding for Myzus persicae clone O genomic sequencing was provided by The Genome Analyses Centre (TGAC) Capacity and Capability Challenge program project CCC-15 and BB/J004553/1 from the Biotechnology and Biological Sciences Research Council (BBSRC) and the John Innes Foundation. 


\section{Authors' contribution}

MD performed analyses, and drafted figures and the manuscript. $\mathrm{AH}$ designed the study, and drafted the manuscript and figures. CB identified key elements from aphid genomes. TB wrote the pipeline employed for database searches. All authors read and approved the final manuscript.

\section{Funding}

This work was supported by a Biotechnology and Biological Sciences Research Council (BBSRC) David Phillips Fellowship to AH (grant number: BB/ N020146/1). CB received funding from the European Research Council (ERC) under the European Union's Horizon 2020 research and innovation programme (grant agreement $n^{\circ} 646625$ ). Toby Baril was supported by the BBSRC South West Biosciences Doctoral Training Partnership (SWBio DTP, training grant reference BB/M009122/1).

\section{Availability of data and materials}

The data used in this study are available in Supplementary material.

\section{Ethics approval and consent to participate}

Not applicable.

\section{Consent for publication}

Not applicable.

\section{Competing interests}

The authors declare that they have no competing interest.

\section{Received: 28 November 2019 Accepted: 8 April 2020}

\section{Published online: 29 June 2020}

\section{References}

1. Ray DA, Feschotte C, Pagan HJT, Smith JD, Pritham EJ, Arensburger P, et al. Multiple waves of recent DNA transposon activity in the bat, Myotis lucifugus. Genome Res. 2008;18:717-28.

2. Chapman JA, Kirkness EF, Simakov O, Hampson SE, Mitros T, Weinmaier T, et al. The dynamic genome of Hydra. Nature. 2010;464:592-6.

3. Muszewska A, Steczkiewicz K, Stepniewska-Dziubinska M, Ginalski K. Cutand-paste transposons in fungi with diverse lifestyles. Genome Biol Evol. 2017:9:3463-77.

4. Yuan Y-W, Wessler SR. The catalytic domain of all eukaryotic cut-and-paste transposase superfamilies. Proc Natl Acad Sci [Internet] 2011;108:7884-7889. Available from: https://doi.org/10.1073/pnas.1104208108.

5. Emmons SW, Yesner L, Ruan K, Katzenberg D. Evidence for a transposon in Caenorhabditis elegans. Cell. 1983:32:55-65.

6. Jacobson JW, Medhora MM, Hartl DL. Molecular structure of a somatically unstable transposable element in Drosophila. Proc Natl Acad Sci. 1986;83: 8684-8.

7. Tenzen T, Matsutani S, Ohtsubo E. Site-specific transposition of insertion sequence IS630. J Bacteriol. 1990;172:3830-6.

8. Smit AFA, Riggs AD. Tiggers and other DNA transposon fossils in the human genome. Proc Natl Acad Sci. 1996;93:1443-8.

9. Robertson HM. The Tc1-mariner superfamily of transposons in animals. J Insect Physiol. 1995;41:99-105.

10. Tudor M, Lobocka M, Goodell M, Pettitt J, O'Hare K. The pogo transposable element family of Drosophila melanogaster. MGG Mol Gen Genet. 1992;232: $126-34$

11. Jarvik T, Lark KG. Characterization of Soymar1, a Mariner element in soybean. Genetics. 1998;149:1569-74.

12. Shao H, Tu Z. Expanding the diversity of the IS630-TC1-mariner superfamily: discovery of a unique DD37E transposon and reclassification of the DD37D and DD39D transposons. Genetics. 2001;159:1103-15.

13. Tellier M, Bouuaert CC, Chalmers R. Mariner and the ITm superfamily of transposons. Microbiol Spectr. 2014;3:1-19.

14. Narayanavari SA, Chilkunda SS, Ivics Z, Izsvák Z. Sleeping Beauty transposition: from biology to applications. Crit Rev Biochem Mol Biol. 2017; 52:18-44.

15. Miskey C, Izsvák Z, Plasterk RH, Ivics Z. The Frog Prince: a reconstructed transposon from Rana pipiens with high transpositional activity in vertebrate cells. Nucleic Acids Res. 2003;31:6873-81.

16. Robertson HM, Zumpano KL. Molecular evolution of an ancient mariner transposon, Hsmar1, in the human genome. Gene. 1997;205:203-17.
17. Miskey C, Mátés L, Sinzelle L, Keller H, Izsvák Z, Ivics Z, et al. The ancient mariner sails again: transposition of the human Hsmarl element by a reconstructed transposase and activities of the SETMAR protein on transposon ends. Mol Cell Biol. 2007:27:4589-600.

18. Deschamps F, Langin T, Maurer P, Gerlinger C, Felenbok B, Daboussi M-J. Specific expression of the Fusarium transposon Fot1 and effects on target gene transcription. Mol Microbiol. 1999;31:1373-83.

19. Tellier M, Chalmers R. Human SETMAR is a DNA sequence-specific histonemethylase with a broad effect on the transcriptome. Nucleic Acids Res. Oxford University Press. 2019;47:122-33.

20. Cordaux R, Udit S, Batzer MA, Feschotte C. Birth of a chimeric primate gene by capture of the transposase gene from a mobile element. Proc Natl Acad Sci. 2006;103:8101-6.

21. Kipling D, Warburton PE. Centromeres. CENP-B and Tigger too Trends Genet. 1997:13:141-5.

22. Casola C, Hucks D, Feschotte C. Convergent domestication of pogo-like transposases into centromere-binding proteins in fission yeast and mammals. Mol Biol Evol. 2008;25:29-41.

23. D'Alençon E, Nègre N, Stanojcic S, Alassoeur B, Gimenez S, Léger A, et al. Characterization of a CENP-B homolog in the holocentric Lepidoptera Spodoptera frugiperda. Gene [Internet]. Elsevier B.V.; 2011;485:91-101. Available from: https://doi.org/10.1016/j.gene.2011.06.007.

24. Smith A, Rutherford K, Benkel B. Characterization of a Tigger1 element from the genome of the American mink (Neovison vison). Gene [Internet]. Elsevier B.V.; 2012;498:164-8. Available from: https:/doi.org/10.1016/j.gene.2012.02.014.

25. Schemberger MO, Nogaroto V, Almeida MC, Artoni RF, Valente GT, Martins $\mathrm{C}$, et al. Sequence analyses and chromosomal distribution of the TCl/ Mariner element in Parodontidae fish (Teleostei: Characiformes). Gene [Internet]. Elsevier B.V.; 2016;593:308-14. Available from: https://doi.org/10. 1016/j.gene.2016.08.034

26. Gao B, Chen W, Shen D, Wang S, Chen C, Zhang L, et al. Characterization of autonomous families of Tc1/mariner transposons in neoteleost genomes. Mar genomics [internet]. The Authors; 2017;34:67-77. Available from: https:// doi.org/10.1016/.margen.2017.05.003.

27. Bouallègue M, Filée J, Kharrat I, Mezghani-Khemakhem M, Rouault JD, Makni $\mathrm{M}$, et al. Diversity and evolution of mariner-like elements in aphid genomes. BMC Genomics BMC Genomics. 2017;18:1-12.

28. Gomulski LM, Torti C, Bonizzoni M, Moralli D, Raimondi E, Capy P, et al. A new basal subfamily of mariner elements in Ceratitis rosa and Other Tephritid flies. J Mol Evol. 2001;53:597-606.

29. Claudianos C, Brownlie J, Russell R, Oakeshott J, Whyard S. maT - a clade of transposons intermediate between mariner and Tc1. Mol Biol Evol. 2002;19: 2101-9.

30. Franz G, Savakis C. Minos, a new transposable element form Drosophila hydei is a member of the Tc1-like family of transposons. Nucleic Acids Res. 1991;19:6646.

31. Moschetti R, Caggese C, Barsanti P, Caizzi R. Intra- and interspecies variation among Bari-1 elements of the melanogaster species group. Genetics. 1998; 150:239-50

32. Clare EL, Barber BR, Sweeney BW, Hebert PDN, Fenton MB. Eating local: influences of habitat on the diet of little brown bats (Myotis lucifugus). Mol Ecol. 2011:20:1772-80

33. Schaack S, Gilbert C, Feschotte C. Promiscuous DNA: horizontal transfer of transposable elements and why it matters for eukaryotic evolution. Trends Ecol Evol. 2010;25:537-46.

34. Thines M. Oomycetes Curr Biol. 2018;28:R812-3.

35. Puzakov M V., Puzakova L V., Cheresiz S V. An analysis of $15630 / T \mathrm{c} 1 /$ mariner transposons in the genome of a Pacific oyster, Crassostrea gigas. J Mol Evol [internet]. Springer US; 2018;86:566-580. Available from: https://doi.org/10. 1007/s00239-018-9868-2.

36. Robertson HM, Soto-adames FN, Walden KKO, Avancini RMP, Lampe DJ. The mariner transposons of animals: horizontally jumping genes. In: Kado C, editor. Horiz gene Transf. Academic P. san Diego; 2002. p. 173-185.

37. Robertson HM. Multiple Mariner transposons in flatworms and hydras are related to those of insects. J Hered. 1997:88:195-201.

38. Jacobs G, Dechyeva D, Menzel G, Dombrowski C, Schmidt T. Molecular characterization of Vulmar1, a complete mariner transposon of sugar beet and diversity of mariner- and En/Spm-like sequences in the genus Beta. Genome. 2004:47:1192-201.

39. Judelson HS. Metabolic diversity and novelties in the Oomycetes. Annu Rev Microbiol. 2017:71:21-39. 
40. Dupeyron M, Singh KS, Bass C, Hayward A. Evolution of Mutator transposable elements across eukaryotic diversity. Mob DNA Mobile DNA. 2019;10:1-14.

41. Hunter DJ, Williams K, Cartinhour S, Herrick G. Precise excision of telomerebearing transposons during Oxytricha fallax macronuclear development. Genes Dev. 1989:3:2101-12.

42. Jahn CL, Doktor SZ, Frels JS, Jaraczewski JW, Krikau MF. Structures of the Euplotes crassus Tec1 and Tec2 elements: identification of putative transposase coding regions. Gene. 1993;133:71-8.

43. Chen $X$, Landweber LF. Phylogenomic analysis reveals genome-wide purifying selection on TBE transposons in the ciliate Oxytricha. Mob DNA [internet]. Mob DNA; 2016;7:1-10. Available from: https://doi.org/10.1186/ s13100-016-0057-9.

44. Doak TG, Doerder FP, Jahn CL, Herrick G. A proposed superfamily of transposase genes: transposon-like elements in ciliated protozoa and a common "D35E" motif. Proc Natl Acad Sci. 1994;91:942-6.

45. Doak TG, Witherspoon DJ, Jahn CL, Herrick G. Selection on the genes of Euplotes crassus Tec1 and Tec2 transposons: evolutionary appearance of a programmed frameshift in a Tec2 gene encoding a tyrosine family sitespecific recombinase. Eukaryot Cell. 2003;2:95-102.

46. Swart EC, Bracht JR, Magrini V, Minx P, Chen X, Zhou Y, et al. The Oxytricha trifallax macronuclear genome: a complex eukaryotic genome with 16,000 tiny chromosomes. PLoS Biol. 2013;11.

47. Smyth JD. Flatworm [internet]. Britannica: Encyclopaedia Britannica, inc.; 2014. Available from: https://www.britannica.com/animal/flatworm.

48. Hand CH, Fautin DG. Cnidarian [Internet]. Encycl. Br: Encyclopaedia Britannica, inc.; 2017. Available from: https://www.britannica.com/animal/cnidarian.

49. Mateo L, González J. Pogo-like transposases have been repeatedly domesticated into CENP-B-related proteins. Genome Biol Evol. 2014;6:2008-16.

50. Negoua AH, Rouault JD, Chakir M, Capy P. Internal deletions of transposable elements: the case of Lemi elements. Genetica. 2013;141:369-79.

51. Banks JA. Selaginella and 400 million years of separation. Annu Rev Plant Biol. 2009;60:223-38.

52. Banks JA, Nishiyama T, Hasebe M, Bowman JL, Gribskov M, DePamphilis C, et al. The Selaginella genome identifies genetic changes associated with the evolution of vascular plants. Science (80- ). 2011;332:960-3.

53. Marshall OJ, Choo KHA. Putative CENP-B paralogues are not present at mammalian centromeres. Chromosoma. 2012;121:169-79.

54. Kersey PJ, Allen JE, Allot A, Barba M, Boddu S, Bolt BJ, et al. Ensembl genomes 2018: an integrated omics infrastructure for non-vertebrate species. Nucleic Acids Res Oxford University Press. 2018;46:D802-8.

55. Stark C, Breitkreutz B-J, Reguly T, Boucher L, Breitkreutz A, Tyers M. BioGRID: a general repository for interaction datasets. Nucleic Acids Res. 2006;34:D535-9.

56. Bastian F, Parmentier G, Roux J, Moretti S, Laudet V, Robinson-Rechavi M. Bgee: Integrating and comparing heterogeneous transcriptome data among species. Lect Notes Comput Sci (including Subser Lect Notes Artif Intell Lect Notes Bioinformatics). 2008;5109 LNBI:124-31.

57. Hruz T, Laule O, Szabo G, Wessendorp F, Bleuler S, Oertle L, et al. Genevestigator V3: a reference expression database for the meta-analysis of Transcriptomes. Adv Bioinforma. 2008;2008:1-5.

58. Combes C. Parasitism: the ecology and evolution of intimate interactions. The Univer. The University of Chicago; 2001.

59. El Baidouri M, Carpentier MC, Cooke R, Gao D, Lasserre E, Llauro C, et al. Widespread and frequent horizontal transfers of transposable elements in plants. Genome Res. 2014;24:831-8.

60. Peccoud J, Loiseau V, Cordaux R, Gilbert C. Massive horizontal transfer of transposable elements in insects. Proc Natl Acad Sci [Internet]. 2017;114 4721-4726. Available from: https://doi.org/10.1073/pnas.1621178114.

61. Altschul SF, Madden TL, Schäffer AA, Zhang J, Zhang Z, Miller W, et al. Gapped BLAST and PSI-BLAST: a new generation of protein database search programs. Nucleic Acids Res. 1997:25:3389-402.

62. Bairoch A, Apweiler R. The SWISS-PROT protein sequence database and its supplement TrEMBL in 2000. Nucleic Acids Res. 2000:28:45-8.

63. Hernandez-Hernandez EM, Fernández-Medina RD, Navarro-Escalante L, Nuñez J, Benavides-Machado P, Carareto CMA. Genome-wide analysis of transposable elements in the coffee berry borer Hypothenemus hampei (Coleoptera: Curculionidae): description of novel families. Mol genet genomics. Springer. Berlin Heidelberg. 2017;292:565-83.

64. Gouveia JG, Wolf IR, Vilas-Boas LA, Heslop-Harrison JS, Schwarzacher T, Dias AL. Repetitive DNA in the catfish genome: rDNA, microsatellites, and Tc1- mariner transposon sequences in Imparfinis species (Siluriformes, Heptapteridae). J Hered. 2017;108:650-7.

65. Dupeyron M, Leclercq S, Cerveau N, Bouchon D, Gilbert C. Horizontal transfer of transposons between and within crustaceans and insects. Mob DNA. 2014;5.

66. Rice $\mathrm{P}$, Ian L, Bleasby $\mathrm{A}$. The European molecular biology open software suite EMBOSS: the European molecular biology open software suite. Trends Genet. 2000;16:276-7.

67. Kumar S, Stecher G, Tamura K. MEGA7: molecular evolutionary genetics analysis version 7.0 for bigger datasets. Mol Biol Evol. 2016;33:1870-4.

68. Edgar RC. MUSCLE: multiple sequence alignment with high accuracy and high throughput. Nucleic Acids Res [Internet]. 2004 [cited 2014 Jul 11];32: 1792-7. Available from: http://www.pubmedcentral.nih.gov/articlerender. fcgi?artid=390337\&tool=pmcentrez\&rendertype=abstract

69. Price MN, Dehal PS, Arkin AP. FastTree 2 - approximately maximumlikelihood trees for large alignments. PLoS One. 2010;5:e9490.

70. Rutherford K, Parkhill J, Crook J, Horsnell T, Rice P, Rajandream M-A, et al. Artemis : sequence visualization and annotation. Bioinformatics. 2000;16: 944-5.

71. Brázda V, Kolomazník J, Lýsek J, Hároníková L, Coufal J, Št'astný J. Palindrome analyser - a new web-based server for predicting and evaluating inverted repeats in nucleotide sequences. Biochem Biophys Res Commun. 2016:478:1739-45.

72. Huerta-Cepas J, Serra F, Bork P. ETE 3: reconstruction, analysis, and visualization of Phylogenomic data. Mol Biol Evol. 2016;33:1635-8.

73. RStudio team, RStudio. RStudio: Integrated development environment for R [Internet]. J. Wildl. Manage. Boston, MA: RStudio, Inc.; 2015. p. 1. Available from: http://www.rstudio.org.

74. Paradis E, Schliep K. Ape 5.0: an environment for modern phylogenetics and evolutionary analyses in R. Bioinformatics. 2019;35:526-8.

\section{Publisher's Note}

Springer Nature remains neutral with regard to jurisdictional claims in published maps and institutional affiliations.
Ready to submit your research? Choose BMC and benefit from:

- fast, convenient online submission

- thorough peer review by experienced researchers in your field

- rapid publication on acceptance

- support for research data, including large and complex data types

- gold Open Access which fosters wider collaboration and increased citations

- maximum visibility for your research: over $100 \mathrm{M}$ website views per year

At $\mathrm{BMC}$, research is always in progress.

Learn more biomedcentral.com/submissions 